\title{
SPECTROSCOPIC DIAGNOSTICS OF PRECESSING DISCS: THE STREAM-DISC IMPACT IN XTE J1118+480 (= KV UMA) IN OUTBURST
}

\author{
C. A. Haswell, ${ }^{1}$ S. B. Foulkes, ${ }^{1}$ D. J. Rolfe ${ }^{2,1}$ J. R. Murray ${ }^{2,3}$ G. H. Tovmassian ${ }^{4}{ }^{4}$ S. V.Zharikov ${ }^{4}$ P. F. L. \\ Maxted,${ }^{5}$ R. North, ${ }^{6}$ P. A. Charles,${ }^{6}$ and R. I. Hynes ${ }^{7,6}$
}

\section{RESUMEN}

Presentamos las trazas de espectrogramas orbitales de XTE J1118+480 tomados en el momento de alcanzar el máximo ell 2000. Muestran un rasgo envolvente de onda S creciendo desde el impacto del chorro en el borde del disco excéntrico en precesión. Comparando las ondas S observadas con las generadas en una simulación SPH, deducimos las fasc's del disco en precesión correspondientes a nuestras observaciones. A partir de ahí inferimos cambios en el período de precesión del disco alrededor del máximo de la explosión: nuestra espectroscopía sugiere un período dr precesión con una duración tan corta como 20 días.

\section{ABSTRACT}

We present orbital trailed spectrograms of XTE J1118+480 taken at the rise to outburst maximum in 2000. These show an evolving S-wave feature arising from the stream impact on the edge of the eccentric precessing disc. By comparing the observed S-waves with those generated in an SPH simulation, we deduce the disc precession phases corresponding to our observations. Hence we infer changes in the disc precession period around the outburst maximum: taken at face value our spectroscopy suggests a precession period temporarily as short as 20 days.

\section{hey Hords: ACCRETION, ACCRETION DISCS - STARS: INDIVIDUAL (XTE J1118+480)}

XTE J1118+480 is a black hole X-ray transient in the Galactic halo. In outburst the optical light curve exhibited is strong 'superhump' modulation, indicating apsidal precession of a non-axisymmetric accretion disc (U'mura of al. 2002).

Torres et al. (2002) published spectroscopy of XTE J1118+48() during outburst, showing that the orbital trailed spectrograms crolve with time. They attributed this to disc precession, but without a detailed model. Zurita et al. (2002) reached similar conclusions from their quiescent spectroscopy of the sistem.

Fig. 1 shows our spectroscopy taken on four night at the risc to the outburst peak. The spectra were phase-folded and binned to produce these trailed spectrograms which show an orbital S-wave which rolves from night to night. On 2000 April 20 the blue side of the S-wave is brighter while on the three other nights the red side is brightcr. The shape of the S-wave also evolves: this is most clearly seen by comparing $\mathrm{H} \delta$ on the two consecutive nights shown

\footnotetext{
${ }^{1}$ Open University, $\mathrm{LK}$.

${ }^{2}$ J. Leicester, UK.

${ }^{3}$ Swinburne Eniversity. Australia.

${ }^{4}$ Instituto de Astrononía, UNAM. Ensanada. México.

5 L. Keele, UK.

"L. Southampton, UK.

${ }^{7} \mathrm{~L}$. Texas at Austin, USA.
}

in the middle two rows of Fig. 1. On 2000 April 12 the $\mathrm{S}$-wave spends more time on the red side of the line profile, traversing to the blue side during the interval phase $0.25-0.50$ and then returning to the red side during the interval $0.50-0.80$; on 2000 April 13 the red-blue traverse is more gradual, and the S-wave reaches its bluest extreme only at phase 0.805 , and then makes a more rapid traverse back to the red side by phase 1.0.

We used the velocity and dissipation data from an SPH simulation in a $q=0.1$ binary to generate the synthetic trailed spectra shown in Fig. 2 (Foulkes et al. $2004^{8}$ ).

By comparing the S-waves in our XTEJ1118+480 trails (Fig. 1) and those of Torres et al. (2002) with the results of our SPH simulation (Fig. 2) we estimated the precession phase ranges compatible with each observed orbital trail. The results are shown in the lower panel of Fig. 3. In some cases, such as our trails on 2000 April 13 (MJD-51000 = 647), the shape of the observed S-wave is clearly defined and is compatible with the simulated trails for only a narrow range of disc precession phase. In other cases, such as our trails on 2000 April $10($ MJD-51000 $=644)$, the observed $\mathrm{S}$-wave is less distinct, and a large range

\footnotetext{
${ }^{8}$ http://physics.open.ac.uk/FHMR/
} 


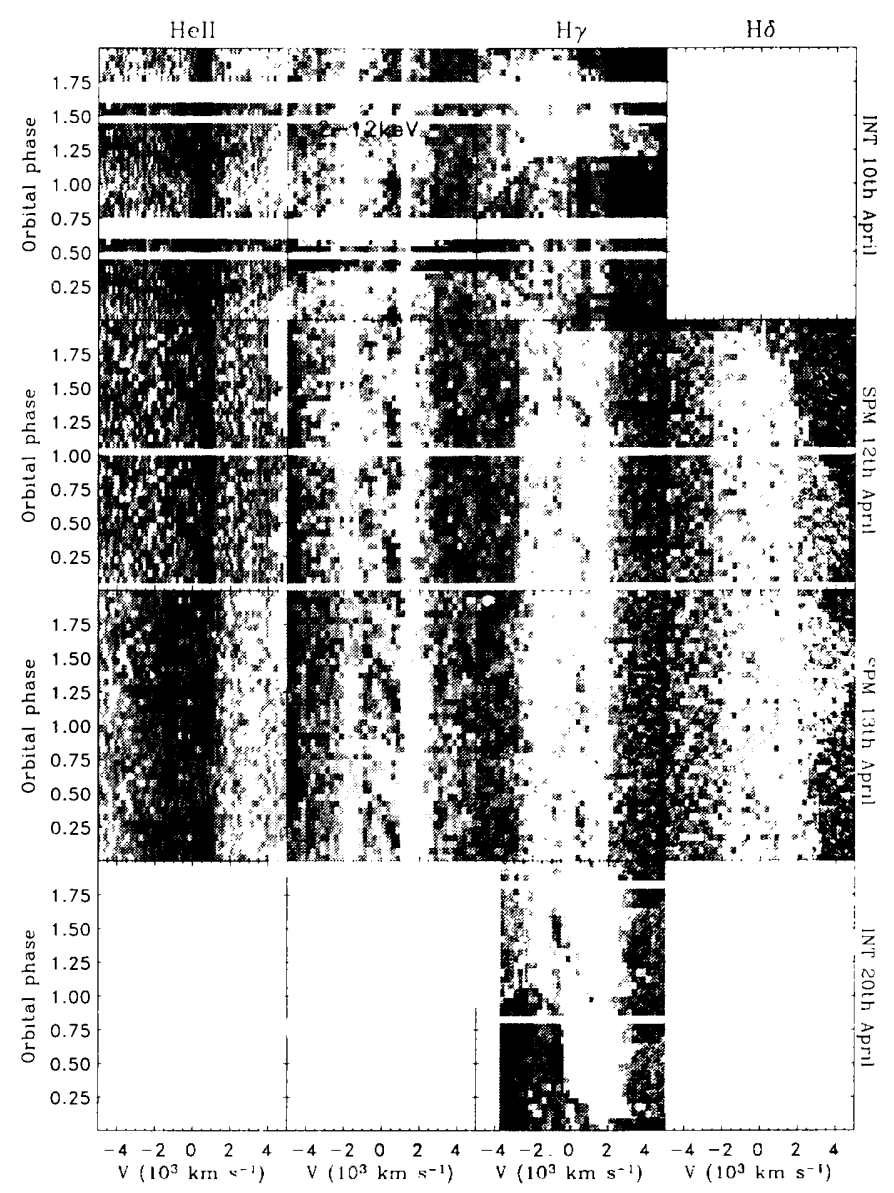

Fig. 1. Trailed spectra of XTE J1118+480 from the INT and SPM telescopes.

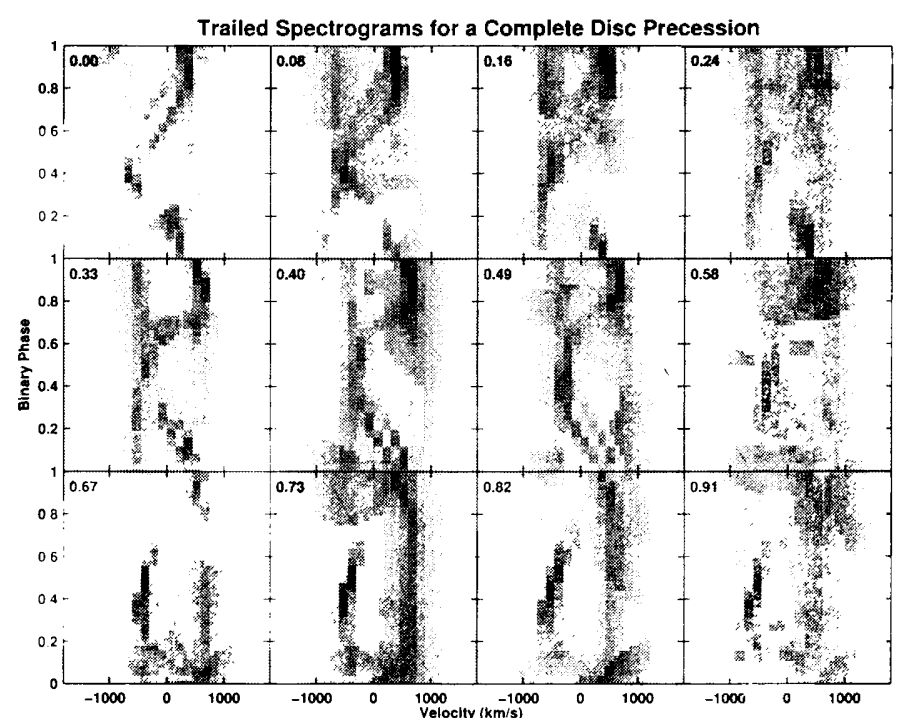

Fig. 2. Low resolution versions of orbital trailed spectra computed from SPH simulations of a precessing disc in a $q=0.1$ binary. The number in the upper left of each panel indicates disc precession phase.

of disc precession phases provide plausible matches. Despite these sometimes large uncertainties in the precession phase on a particular night, it is

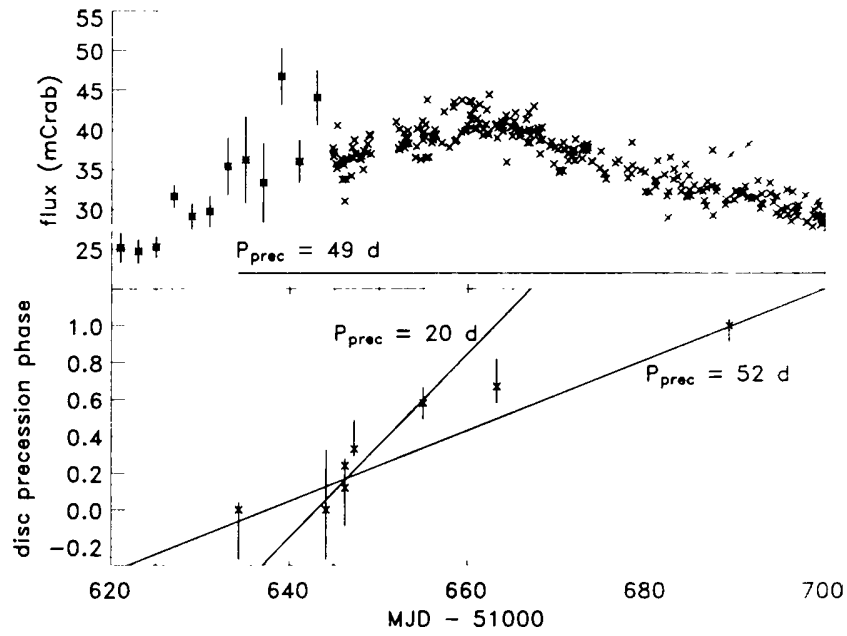

Fig. 3. Upper panel: RXTE/USA X-ray ditid. and the baseline of Uemura et al. 2002 photometry. Lower panel: Disc precession phase estimates. The straight lines illustrate 52 day and 20 day precession periods for comparison.

clear that no single straight line provides a good fit to the points in the lower panel of Fig. 3. i.e. the disc precession period changes. I'cmura it al. (2002) reported changes in the superhump period, which are consistent with our cstimated precession phases. Osaki's empirical relationship between disc precession period, disc radius. and mass ratio (e.g. Mineshige, Hirose \& Osaki 1992) is an approximation: Lubow (1992) deducerl that the precession period also depends on the second time derivative of the eccentric mode strength. so we should expect changes in the precession period as the outburst approaches its peak. Taken at face value, our estimated phases imply a precession period as short as $\sim 20$ days around MIJD 51650 .

The encouraging match between some of the simulated and observed trails suggests this may prove a much more powerful diagnostic of the drnamic: of tidally stressed precessing discs than photonctry alone. Spectroscopy of future X-ray transients in outburst might, for example, allow us to infer their mass ratio and other system parameters.

\section{REFERENCES}

Foulkes, S.B., Haswell, ('A.. Murray: J.R., Rolfe, D.J 2004, MNRAS. in press: astro-ph (0401.xxx.

Lubow, S. 1992, Ap.J. 401, 317l.

Mineshige, S., Hirose, M.. \& Osaki, Y. 1942. P.l.i.l. 1. L 15

Murray. J.R. 2004. these proceedings

Torres, M. A. P. '(t al. 20(0)2. Ip.], 5(i) 1.2.3

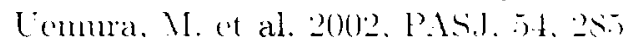

Zurita, ('. et al. 20(1)2, MNR AS, 333, 7.1 